\title{
Cardiac troponin: an emerging cardiac biomarker in animal health
}

\author{
Vishal V. Undhad, Dhaval T. Fefar, B. M. Jivani, Hemant Gupta, Dineshbhai J. Ghodasara, \\ Bholanath P. J oshi, Kantibhai S. Prajapati \\ Department of Veterinary Pathology \\ College of Veterinary Science and Animal Husbandry, Anand Agriculture University, Anand-388001 \\ Corresponding author: Dhaval Tribhovanbhai Fefar, E-mail: fdhaval@gmail.com \\ Received: 23-01-2012, Accepted: 11-02-2012, Published Online: 24-05-2012 \\ doi: $10.5455 /$ vetworld.2012.508-511
}

\begin{abstract}
Analysis of cardiac troponin I (cTn I) and T (cTnT) are considered the "gold standard" for the non-invasive diagnosis of myocardial injury in human and animals. It has replaced traditionally used cardiac biomarkers such as myoglobin, lactate dehydrogenase (LDH), creatine kinase (CK) and CK-MB due to its high sensitivity and specificity for the detection of myocardial injury. Cardiac troponins are proteins that control the calcium-mediated interaction between actin and myosin, allowing contraction at the sarcomere level. Concentration of the cTn can be correlated microscopic lesion and loss of immunolabeling in myocardium damage. Troponin concentration remains elevated in blood for 1-2wks so that wide window is available for diagnosis of myocardial damage. The cTn test has $>95 \%$ specificity and sensitivity and test is less time consuming (10 to 15 minutes) and less costly (INR 200 to INR 500).

Keywords: Cardiac troponin C, I and T, myocardium, cardiac biomarkers
\end{abstract}

To cite this article :

Undhad VV, Fefar DT, Jivani BM, Gupta H, Ghodasara DJ, Joshi BP, Prajapati KS (2012) Cardiac troponin: an emerging cardiac biomarker in animal health, Vet World 5(8):508-511. doi: 10.5455/vetworld.2012.508-511

\section{Introduction}

There are so many causes due to which myocardium damage occurs in animals like infectious diseases, toxic chemicals and drugs, nutritional deficiencies, trauma etc. Each and every year we loose lot of money, time and man power due to miss leading diagnosis of heart diseases or problems. There are some external tools (cardiac auscultation,radiography, ECG and echocardiography) and some traditional biomarkers (Myoglobin, CK, CK-MB, LDH and SGOT) for routine diagnosis of heart diseases but all of these have some limitations as they do not posses $100 \%$ specificity and sensitivity to heart. They also increase in other disease condition and they do not able to identify minor myocardial damage. Troponin is a complex of three regulatory proteins that are integral to muscle contraction in skeletal and cardiac muscle, but not in smooth muscle. Troponins are not found to such a significant extent in other tissues, although there is an analogous protein, calmodulin in smooth muscle [1]. Cardiac troponin I (cTnI) and cardiac troponin $\mathrm{T}(\mathrm{c} \operatorname{Tn} \mathrm{T})$ are uniquely expressed in the myocardium and have been widely recognized as highly sensitive and specific serum markers for the noninvasive diagnosis of increased myocyte permeability or necrosis. First cTnI was identified in 1987 and later cTnT was identified in 1989 as biomarkers of cardiac cell death [2].

\section{Biochemistry of troponin}

There are three types of troponin those are troponin $\mathrm{C}$, I and $\mathrm{T}$. Troponin $\mathrm{C}$ is the $\mathrm{Ca}^{2+}$-binding component of troponin while the troponin $\mathrm{I}$ is responsible for inhibition of the actomyosin ATPase and troponin $\mathrm{T}$ is interacts with tropomyosin and plays an important role in regulation of muscle contraction. Troponin C, I, and T have two (Fast \& Slow skeletal), three ( 2 unique for skeletal muscle and 1 for cardiac muscle) and multiple isoforms respectively. Molecular mass of troponin C, I and T were 18, 22.5 and $37 \mathrm{kd}$ respectively. Troponin $\mathrm{C}$ is of little value for the specific diagnosis of myocardial injury because of extracardiac expression in slow skeletal muscle fibers. In contrast troponin $\mathrm{T} \& \mathrm{I}$ are ideally suited as serodiagnostic markers for myocardial injury since the heart \& extracardiac muscle express distinct isoforms which are encoded by different genes [3]. 


\section{Physiology of troponin}

In the 1960's it was hypothesized that the contraction of striated muscle is regulated by a special protein complex located on actin filaments this complex was called native Tropomyosin [4]. This native tropomyosin consists of two parts tropomyosin and troponin [5]. Tropomyosin is a single long protein strand that winds around the actin filament while troponin is a globular complex of three proteins and is found in clumps around the Tropomyosin protein.

In animals without induced myocardial damage cTnT is undetectable $(<0.01 \mathrm{ng} / \mathrm{ml})$ in serum [6]. The cTnI concentration in Dog $0.02 \mathrm{ng} / \mathrm{ml}$ and Cat 0.04 $\mathrm{ng} / \mathrm{ml}$ [7] while Cows $\leq 0.04 \mathrm{ng} / \mathrm{ml}[8]$ and Horses $\leq$ $0.015 \mathrm{ng} / \mathrm{ml}$ [9]. Human studies revealed that levels of cTnT in the neonates were significantly higher than those in children of older age [10]. Younger broiler chicks exhibited a significantly higher cTnT concentration than the older chicks, thereby supporting the fetal origin hypothesis of this marker protein in the younger age group. In the rat, an age and gender dependent variation in serum cTnI was found. Male rats aged six and eight months had a 10-fold greater serum cTnI than age matched females and threemonth-old rats [11]. In healthy cow age, body weight, lactation, and pregnancy status had no effect on baseline cTnI concentration.

\section{Specificity and sensitivity of troponin}

The cTnI and cTnT are $40 \%$ and $10-30 \%$ structurally distinct from the skeletal troponin I and troponin T isoforms respectively [12]. It is imperative to recognize that increase in the concentration of serum cardiac troponins indicate myocardial damage but do not indicate mechanism [13]. Both cTnT and cTnI have been used as biomarkers to detect druginduced cardiac injury in humans and animals [14]. The cTnT has also been reported to be an independent predictor of early postoperative cardiovascular complications following non-cardiac surgery. Most intracellular cTnI and cTnT is bound to the myofibrils in the cardiac myocyte however a small percentage exists in a cytosolic pool (6-8\% of cTnT and 3-4\% of $\mathrm{cTnI}$ ) with the majority of the remaining troponin found in the sarcomere [15]. After myocardial damage cardiac troponin concentration increase within 3-8 hrs and reach pick concentration at 12-24 hrs and remain elevated for 1-2 wks (Table-1) which provide wide windows available for diagnosis of myocardial damage.

Normally, cTnT concentration is $10.2 \mathrm{mg} / \mathrm{g}$ myocardial tissue which is 2 times than cTnI in myocardium where as cTnI concentration is 13 times more than CK-MB in myocardium so ultimately cTnT concentration is becomes 26 times more than CK-MB in myocardium so even micro necrosis in myocardium than with help of cTnT can be identified. A good relationship between cTnI concentration and the extent and severity of myocardial injury as shown by histopathologic evaluation of cardiac tissue in people and laboratory animals has been observed [16]. Minimum serum concentrations of $1.3 \mathrm{ng} / \mathrm{ml}$ for cTnI and $0.35 \mathrm{ng} / \mathrm{ml}$ for cTnT are necessary for myocardial cell injury to be detected histologically [17]. Loss of cTnI is also identified by immunolabelin even in the absence of histologic proof of myocardial necrosis or degeneration. Immunolabeling for cTnI was more sensitive than routine $\mathrm{H}$ \& $\mathrm{E}$ staining for the recognition of peracute myocardial necrosis in both experimental animals and humans [18].

Table-1. Comparison of various cardiac biomarkers with cTn regards to cardiac injury

\begin{tabular}{lccc}
\hline Cardiac Marker & $\begin{array}{c}\text { Increase } \\
\text { (hours) }\end{array}$ & $\begin{array}{c}\text { Peak } \\
\text { (hours) }\end{array}$ & $\begin{array}{c}\text { Normalization } \\
\text { (Days) }\end{array}$ \\
\hline CK & $3-12$ & $12-24$ & $3-4$ \\
CK-MB & $3-12$ & $12-25$ & $2-3$ \\
CK-MB mass & $2-6$ & $12-24$ & 3 \\
Myoglobin & $2-6$ & $6-12$ & 1 \\
cTnl & $3-8$ & $12-24$ & $7-10$ \\
cTnT & $3-8$ & $12-96$ & $7-14$ \\
\hline
\end{tabular}

\section{Causes of elevated troponin concentration}

Various scientists carried out studies in different species of animals in cardiac diseases condition and concluded that increase in the level of cardiac troponin is associated with cardiac injuries. The level of cTn in dog is increased in pericardial effusion, cardiac contusion, cardiomyopathy, babesiosis, gastric dilatation and volvulus (GDV), ehrlichiosis and chronic mitral valve disease while in cat hypertrophic cardiomyopathy, in horse valvular disease and ventricular tachycardia, in cattle idiopathic pericarditis and traumatic reticulopericarditis (TRP), in calf foot and mouth disease (FMD) and endotoxemia and in poultry ascites.

\section{Causes of elevated troponin in renal failure}

The cTnT $(53 \%)$ remains elevated to a higher degree than cTnI $(17 \%)$ in patients with chronic kidney disease (CKD) in the absence of clinical acute myocardial necrosis (19). However in most end stage renal disease (ESRD) patients without acute myocardial ischaemia, cTnT is only slightly elevated with a majority of the results below $1.0 \mathrm{ng} / \mathrm{ml}$ and concentrations above $3.0 \mathrm{ng} / \mathrm{ml}$ are extremely rare 
(20). The cTnT assay is so sensitive that it detects subclinical myocardial cell injury whereas the cTnI assays are less sensitive (20). The cTnI is superior to cTnT for the diagnosis of myocardial ischaemia in patients with renal failure. Several mechanisms have been proposed for this increase troponin in renal diseases, although no definite explanation exists.

\section{Causes of false positive troponin results}

If the animal's clinical pictures for myocardial damage do not match an elevated troponin result, the laboratory should suspect a false positive troponin value caused by analytical interferences with this assay. These analytic interferences include heterophile antibodies, human anti-animal antibodies, autoantibodies, rheumatoid factor, fibrin clots, hemolysis interference, high concentration of AKP, immunocomplex formation and analyzer malfunction. In general, analytical interferences resulting in false positive troponin results are associated with a specific manufacturer's troponin assay and are not encountered in all troponin assays [21].

\section{Tests for measurement of cardiac troponin}

As the homology between troponins is about 95\% among mammals, commercial diagnostic kits designed for use in humans also provide excellent results in other animals including poultry [6]. The Cardiac isoforms of troponin I (cTnI) and troponin T (cTnT) can be specifically recognized by monoclonal or polyclonal antibodies which do not cross react with the skeletal muscle isoforms [22]. For estimation of cardiac troponin sample should be collected whole Blood, Serum or Plasma and anticoagulant used either EDTA or heparin as per the manufactured instruction. Because of international patent restrictions, only one assay for cTnT is available from a single manufacturer (Roche Diagnostics) with uniform cutoff concentration. In contrast, at least 18 different commercial assays for cTnI are available with variation in the cutoff concentrations for the definition of an abnormal concentration of cTnI [23]. Studies have shown that troponin I results may vary by a factor of 100 fold from one assay and manufacturer to another. The reason for some variability in cTnI results is that cTnI is susceptible to proteolytic degradation leading to the appearance in serum of a wide diversity of peptides with different stabilities. Some cTnI epitopes remain unaltered while other epitopes are lost or altered in the degradation process. In addition, the antibody used by a particular manufacturer may be directed against different epitopes of troponin I, which result in assay- to-assay variation in detected levels of cTnI.

\section{References}

1. Winder, S. J. and Walsh, M. P. (1993). Smooth muscle calponin. Inhibition of actomyosin MgATPase and regulation by phosphorylation. J Biol Chem. 265 (17):10148-55.

2. Katus, H. A.; Remppis A. and Looser, S. (1989). Enzyme linked immune assay of cardiac troponin T for the detection of acute myocardial infarction in patients. JMol Cell Cardiol. 21:1349-1353.

3. Baldwin, A. S.; Kittle, E. L. W. and Emerson, C. P. (1985). Structure, evolution, and regulation of skeletal muscle troponin I gene. Proc Natl Acad Sci USA. 82:8080-8084.

4. Ebashi, S.; Kodama, A. and Ebashi, F. (1968). Troponin I preparation and physiological function. $J$. Biochem. 64:465-477.

5. Bailey, K. (1946). Tropomyosin: a new asymmetric protein component of muscle. Nature. 157:368-369.

6. O'Brien, P. J.; Landt, Y. and Ladenson, J. H. (1997). Differential reactivity of cardiac and skeletal muscle from various species in a cardiac troponin I immunoassay. Clin. Chemistry. 43:2333-2338.

7. Sleeper, M. M.; Cliffoer, C. A. and Laster, L. L. (2001). Cardiac troponin I in the normal dog and cat. $J$ Vet Intern Med. 15:501-503.

8. Jesty, S. A.; Sweeney, R. W. and Dolente, B. A. (2005). Idiopathic pericarditis and cardiac tamponade in two cows. JAm Vet Med Assoc. 226:1555-1558.

9. Begg, L. M.; Hoffmann, K. L. and Begg, A. P. (2006). Serum and plasma cardiac troponin I concentrations in clinically normal Thoroughbreds in training in Australia. Aust Vet J84:336-337.

10. Clark, D.; De Morjana, H.; N. and Tal, R. (2001). Biochemical and immunological properties if human cardiac troponin I fragments. Biotechnol Appl Biochem. 33:107-115.

11. O'Brien, P. J.; et.al. (2006). Cardiac troponin I is a sensitive, specific biomarker of cardiac injury in laboratory animals. Laboratory Animals. 40:153-171.

12. Mair, J. (1997). Cardiac troponin I and troponin T: are enzymes still relevant as cardiac markers? Clin. Chem. Acta. 257:99-115.

13. Alpert, J. S.; Thygesen, K.; Antman, E. and Bassand, J. P. (2000). Myocardial infarction redefined-a consensus document of The Joint European Society of Cardiology/American College of Cardiology Committee for the redefinition of myocardial infarction. J Am Coll Cardiol. 36:959-69.

14. O'Brien, P. J. (2008). Cardiac troponin is the most effective translational safety biomarker for myocardial injury in cardiotoxicity. Toxicol. 245:206-218.

15. Bhayana, V. and Henderson, A. R. (1995). Biochemical markers of cardiac damage. Clin. Biochem. 28:1-29.

16. Hasiæ, S.; Kiseljakoviæ, E. and Jadriæ, R.(2003). Cardiac troponin I: the gold standard in acute 
myocardial infarction diagnosis. Bosn $J$ Basic Med Sci. 3:41-44.

17. Bertsch, T.; Bleuel, H.; Aufenanger, J. and Rebel, W. (1997). Comparison of cardiac troponin I and cardiac troponin $\mathrm{T}$ concentrations in peripheral blood during orciprenaline induced tachycardia in rats. Exp. Toxic. Pathol.49:467-468.

18. Recai, T.; et.al. (2008). Determination of cardiac troponin I in the blood and heart of calves with foot and mouth disease. J. Vet. Diagn. Invest. 20:598-605.

19. Kontos, M. C.; Garg, R. and Anderson, F. P. (2005). Outcomes in patients admitted for chest pain with renal failure and troponin I elevations. Am. Heart J. 150:674-680.

20. Lowbeer, C. (2007). Cardiac troponin $\mathrm{T}$ in clinical and experimental studies. M.V.Sc thesis submitted to Department of Laboratory Medicine, Karolinska University, Karolinska Institutet, Stockholm, Sweden.

21. Gifford L. et.al. (2006). False Positive Cardiac Troponin Results in Patients Without Acute Myocardial Infarction. Labmedicine.37(9):546-550.

22. Katus, H. A, et.al. (1995). Cardiac troponin T in end stage renal diseases patients undergoing chronic maintenance hemodialysis (Letter). Clin. Chem. 41:1201-1202.

23. Tate, J. R.; Badrick, T.; Koumantakis, G.; Potter, J. M. and Hickman, P. E. (2002). Reporting of cardiac troponin concentrations. Clinical Chemistry. 48:2077-2080. 